\title{
The Comparative Research on the Location Technology of Wireless Sensor Networks
}

\author{
Jijun ZHAO ${ }^{1,2}$, Hua LI ${ }^{1,3}$, Xin ZHAO ${ }^{1}$ \\ College of Information \& Electronic Engineering, Hebei University of Engineering, Handan, China \\ E-mail:zhaojijun@china.com,lihua9896@163.com \\ Received July 17, 2009; accepted August 3, 2009
}

\begin{abstract}
Wireless Sensor Network (WSN) which is composed of lots of self-organizational intelligence nodes has become new technology of information acquisition and processing. Location technology is one of the key technologies in WSN. There are many kinds of location algorithms currently and the research to performance evaluation and applicability analysis of algorithms has fundamental significance. For this reason, the paper firstly elaborated the performance evaluation indexes of location algorithms, and analyzed the location principles, characteristics and current fundamental problems to typical and some new location algorithms, and then analyzed and compared some typical location algorithms according to the location accuracy, node density and hardware requirement, obtaining the sphere of application of these algorithms and pointing out the problems which need to be solved in WSN currently.
\end{abstract}

Keywords: Wireless Sensor Networks, Location Technology, Location Algorithm, Performance Evaluation

\section{1. 引言}

无线传感器网络就是由部署在监测区域内大量的 微型传感器节点组成, 通过无线通信方式形成的一个 多跳的自组织的网络系统，其目的是协作地实时监 测、感知和采集网络分布区域内的各种环境或监测对 象的信息, 并对这些信息进行处理, 获得详尽而准确 的信息, 再传送给需要这些信息的用户。

定位技术是无线传感器网络的关键技术之一, 在 无线传感器网络中, 确定事件发生的位置或锁定获取 消息的节点的位置是无线传感器网络面向实际应用的 前提, 同时节点位置信息在目标追踪、辅助路由、网 络管理等许多方面也具有重要的作用[1]。目前已有多 种基于测距(Range Based)的定位算法, 能实现相对精 确的定位功能, 但功耗高、硬件限制大。无需测距 (Range Free)的定位算法, 硬件限制低, 但定位粗鋉, 最近也出现了许多在原有定位算法基础上衍生或改进 的新生定位算法。但各种算法均有其优缺点和不同的 适用范围，如何对一个算法进行评价，该算法适用于

项目资助: 河北省教育厅科研计划项目(2008110); 邯䣋市科学技术 研究与发展计划项目(0821103041-2).
什么样的环境成为文章的研究重点, 对此文章首先阐 述了定位算法的性能评价指标, 对典型定位算法及新 生算法的定位原理、特点和存在的基本问题做了系统 的分析, 在此基础上根据定位精度、节点密度和硬件 要求等主要性能评价指标对常用的典型定位算法进行 了性能分析对比, 得出其适用范围, 并指出了 WSN 定 位技术当前需要解决的问题。

\section{2. 节点定位的概念}

无线传感器网络节点的定位是指自组织的网络通 过特定的方法确定节点位置 (节点坐标) 的过程。这 种定位分为两种：自身定位和目标定位，节点自身定 位是确定网络中节点的自身坐标位置的过程, 目标定 位是确定网络覆盖范围内一个事件或者一个目标的坐 标位置，自身定位是目标定位的基础[2]。

在传感器网络节点自身定位中, 根据节点是否已 知自身的位置, 把节点分为信标节点(beacon node)和未 知节点(unknown node)两类。信标节点在网络中所占的 比例很小, 可以通过携带 GPS 定位设备和人工布设等 手段获得自身的位置。除了信标节点外, 其他传感器 
节点就是未知节点, 它们必须依靠信标节点, 通过合 适的定位算法来确定自身位置。目标定位是以位置已 知的节点作为参考节点, 进而确定事件或目标在网络 中的位置。无线传感器网络定位技术就是要求开发适 合网络特征、节能、高效的定位算法。

\section{3. 定位算法主要性能评价指标}

WSN 定位算法的性能直接影响其可用性, 对它们 评价标准的研究是一个重要的问题。下面为定位算法 的几个主要的定性评价标准。

1) 定位精度:定位精度是评价定位技术的首要指 标，一般用误差值与节点无线射程的比例表示。也有 部分定位系统将二维网络部署区域划分为网格, 用网 格的大小表达定位精度, 如微软的 RADAR。

2) 节点密度:在 WSN 中, 定位算法对节点密度有 不同的要求, 节点密度越大网络费用就越大, 并会带 来节点间通信冲突问题。许多定位算法的精度受节点 密度的影响, 如 DV-Hop 算法一般在节点密集部署的 情况下定位。

3) 信标节点密度:信标节点定位通常依赖人工部 署或 GPS 实现。人工部署的方式不但受网络部署环境 的限制，还严重制约了网络和应用的可扩展性。使用 GPS 定位会提高信标节点的费用。因此, 信标节点密 度也是评价定位算法性能的指标之一。

4) 容错能力和自适应能力:定位算法在真实应用 场合中常会有以下问题 : 外界环境中存在严重的多径 传播、衰减、通信盲点、非视距等问题; 节点失效问 题; 节点间的距离或角度测量误差问题。因此, 定位 算法的软、硬件必须具有通过自动调整纠正错误、适 应环境、减小各种误差影响, 以提高定位精度的容错 能力和自适应能力。

5) 定位功耗: 在定位过程中所需的功耗有计算 量、通信开销、存储开销等。由于传感器节点电池能 量有限, 因此在保证定位精度的前提下, 需要尽量低 的功耗。

6) 覆盖率:即可实现定位的未知节点与未知节点 的比例 在 WSN 中可能会有一些不可达到或连通度极 低的未知节点, 实现尽可能多的未知节点的精确定位 是定位算法的目标之一。

7) 规模:不同的定位算法可以实现不同范围的节 点定位。在一定的基础设施或一段时间内实现定位目 标的数量也是一个评价指标。
8) 代价:其包括时间代价, 即系统安装时间、配置 时间、定位所需时间，空间代价，即定位算法所需基 础设施和网络节点的数量、硬件尺寸等, 资金代价, 即实现一种定位算法的基础设施 节点设备的总费用。

以上为 WSN 定位算法的 8 个主要性能评价指标, 它是评价算法性能优劣的标准，也是其设计和实现的 优化标准。同时，这些性能指标也相互关联，须根据 具体需求做出权衡, 选择适合具体应用环境和定位要 求的算法。

\section{4. 节点定位技术}

目前对定位算法的分类有多种方法 , 其中最常用的 分类方法为从是否需要测量节点间实际距离和角度进 行分类，进而得出基于测距 (Range-Based) 的定位算 法和无需测距 (Range-Free) 的定位算法两大类。近年 也出现了许多新颖的定位算法和思路，包括一些典型定 位算法的衍生算法，在此也对其进行系统的阐述 $[3,4]$ 。

\section{1. 基於测距的定位算法介绍}

基于测距的基本定位算法有三边定位法，三角定 位法，极大似然估计法。在这些基本定位算法的基础 上出现了许多新生算法。

AH Los 定位算法为基于三边定位法的迭代定位 算法, 它将已被定位的未知节点升级为信标节点来解 决信标节点的稀疏问题。但会造成误差累加，相对于 测距精度, 该算法的定位精度降低一个数量级。

在 AH Los 定位算法的基础上, 还出现了 N-hop Multilateration Primitive 定位算法。它给出了判定节点 是否可参与 Collaborative Multilateration 的充分条件, 再使用卡尔曼滤波技术循环定位求精, 减小了误差积 累。试验显示, 该算法的定位精度可达 $3 \mathrm{~cm}[5]$ （节点 测距误差为 $1 \mathrm{~cm}$ ，信标节点比例为 $20 \%$ ）。

Cooperative Ranging 和 Two-phase Positioning 定位算法为循环求精的算法。该算法可以减少测距 误差对定位的影响。实验显示, Cooperative Ranging 算法定位精度可达到 $5 \%$ ，测距误差为 $5 \%$ 。而 Two-phase Positioning 算法在信标节点比例为 5\%、 测距误差为 $5 \%$ 、网络连通度为 7 的条件下, 定位精 度约为 $33 \%[6,7]$ 。

Generic Localized Algorithms 定位算法指定了末 知节点位置估算并升级为信标节点的条件，以减少误 
差累计。试验显示, Generic Localized Algorithms 算法 在信标节点定位误差为 $10 \%$ 、信标节点比例为 $20 \%$, 测距误差在 $25 \%$ 的条件下，定位精度为 $40 \%$ [8]。

新生算法均是在三个基本算法的基础上, 并在一 定的信标节点密度, 测距精度等前提下, 利用循环求 精，节点升级的办法在一定程度上提高了定位精度。

在以上基于测距（Range-Based）的定位技术中， 测距是定位的前提，测距即通过特定方法得到节点间 的实际距离。定位过程分为测距阶段, 定位计算阶段 和循环求精阶段, 其中测距阶段需要通过信号传播速 度、信号衰减和辅助硬件设施等计算得出, 在此阶段 极易产生测距误差，而定位计算阶段一般通过三边、 三角或极大似然定位算法直接计算, 产生误差较小, 可见测距精度在很大程度上决定了定位精度。在此详 细介绍几种典型的测距法, 分析其基本特点和存在的 问题，在此基础上研究其适用性。

\section{2. 测距法}

\subsubsection{TOA (T ime To A rrival) 测距法}

在 TOA 测距法中, 已知信号的传播速度, 根据信 号的传播时间来计算节点间的距离, 然后利用三边定 位法计算出节点的位置。若电波从信标节点到未知节 点的传播时间为 $\mathrm{t}$, 电波传播速度为 $\mathrm{v}$, 则信标节点到 未知节点的距离为 $t^{*} v$ 。

由测距原理可见 TOA 测距法简单。收发两节点 都需要记时，从而得知信号传播时间，信号的传播速 度很快，时间测量上的很小误差可能导致很大的测距 误差, 因此测距精度取决于时钟精确度, 这就要求节 点具有非常精确的同步时钟,一般有较高的测距精度。

\subsubsection{TDOA (T ime Difference Of A rrival) 测距法}

在 TDOA 测距法中，一般在节点上安装超声波收 发器和 RF 收发器, 发射节点同时发射两种不同传播速 度的无线信号到接收节点, 接收节点根据两种信号的 到达时间差以及这两种信号的传播速度, 计算两个节 点之间的距离。如图 1 , 利用射频信号和超声波信号, 接收节点记录两种信号到达的时间 $\mathrm{T}_{1}, \mathrm{~T}_{2}$ 已知无线射 频信号和超声波的传播速度为 $\mathrm{C}_{1}, \mathrm{C}_{2}$ 那么两点之间的 距离为:

$$
\left.\left(T_{2}-T_{1}\right) * S \quad \text { (其中 } S=\frac{c_{1} c_{2}}{c_{1}-c_{2}}\right)
$$

TDOA 测距精度高, 测距法要求节点增加声波或

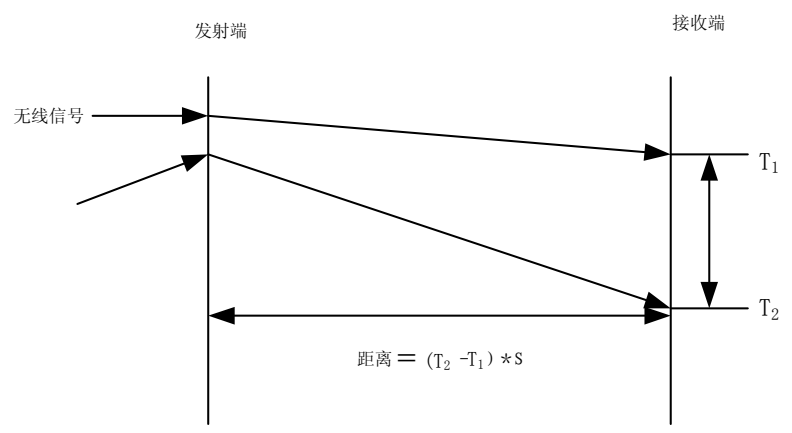

圖 1. TDOA 測距原理圖.

Figure 1. The schematic diagram of TDA ranging algorithm.

超声波收发器，同时需要精确的时钟记录两种信 号的到达时间差，因此测距精度取决于时钟精度，并 且受到超声波或声波的传播距离的限制，受多途、混 响效应影响，不能穿透障碍物。

\subsubsection{AOA (Angle Of Arrival) 测距法}

在 $A O A$ 的测距中, 需要首先给节点配备超声波接 收机或特殊天线。接收节点利用多个超声波接收机或 天线阵列接收发射节点的信号，根据不同节点接收信 号的角度判断信号到达方向，从而计算接收节点和发 射节点之间的相对方位或角度，再通过三角测量法计 算出节点的位置。

基于 $A O A$ 的定位不仅能确定节点的坐标, 还可确 定节点的方位信息。仿真表明，在平均节点密度为 10.5 , 通信距离为 $32 \mathrm{~m}$ 的条件下, 导标节点比例为 $20 \%$; 角度测量误差为 $5^{\circ}$ 时，平均定位误差约为 $30 \%$ [5]（相对通信距离）。

$A O A$ 测距法中每个节点要安装昂贵的天线阵列和 超声波接收器，在硬件尺寸和功耗上要求很高。且易 受外界环境影响。

4.24. RSSI (Received Signal Strength Indicator) 测距法

在 RSSI 测距法中，发射节点的发射信号强度已 知，接收节点记录接收到信号的强度，计算出信号的 传播损耗，再利用信号传播的理论模型或经验模型将 传输损耗转化为距离。如常用的理论衰减模型：

$$
P_{r}(d)=P_{t} G_{t} G_{r} \lambda^{2} /(4 \pi)^{2} d^{2} L
$$

其中 $P_{t}$ 为发射机功率; $P_{r}(d)$ 是在距离 $d$ 处的接收 功率 ; $G_{r} 、 G_{t}$ 分别是发射天线和接收天线的增益; $d$ 是距离; $L$ 为与传播无关的系统损耗因子; $\lambda$ 是波长。 
无线电收发器是 WSN 节点已有的资源, 不需要添 加额外的硬件。故其是一种低功耗，廉价的测距技术。 但在实际使用中，易受湿度、温度、障碍物的影响， 产生多径传播、反射、非视距等现象, 它们都会对相 同距离产生显著不同的传播损耗，最多可造成 \pm 50\% 的测距误差[9]。

由以上测距法可见, 基于测距的定位方法, 除 RSSI 外普遍具有较高的定位精度, 都需要测量与三个信标 节点的距离进而定位, 其中 TOA、TDOA、AOA 测距 法均对节点硬件有较高的要求, 均较易受环境影响。 因此基于测距的定位技术适合在需要较高定位精度且 小规模内应用。

\section{3. 无需测距的定位算法}

\subsection{1. 质心算法}

质心算法的基本思想是 : 通过未知节点以所有在 其通信范围内的所有信标节点的通信区域重叠所组成 多边形的几何质心作为自己的估计位置。定位过程 为: 信标节点周期性向邻居节点广播信标分组, 信标 分组中包含信标节点的标识号和位置信息。当未知节 点接受到来自不同信标节点的信标分组数量超过某一 个门限 $k$ 或接收一定时间后, 就确定自身位置为这些 信标节点所组成的多边形的质心。

质心算法定位原理简单，算法易实现，计算量小， 且完全基于网络的连通性, 无需信标节点和未知节点 之间的协调，但用质心作为实际位置本身就是一种估 计, 这种估计的精度和信标节点的密度以及分布均匀 度有很大关系: 密度越大, 分布越均匀, 定位精度越 高。另外与节点的无线信号传播模型的规则程度有 关，越接近球型精度越高。

\subsubsection{APIT 算法}

在 APIT 算法中, 未知节点从所有能与它通信的信 标节点中任选 3 个节点, 测试它是在这 3 个信标节点 所组成的三角形内部还是外部, 然后再选择另外 3 个 信标节点进行同样的测试, 直到穷尽所有的组合或者 达到所需的精度。最后, 未知节点将包含自己的所有 三角形的相交区域的质心作为自己的估计位置。如图 2(a)所示, $\triangle A O F$ 区域就是所有被节点 $s$ 监听到的包含 $\mathrm{s}$ 的三角形的相交区域, 它的重心被认为是节点 $\mathrm{s}$ 的位 置。(其中 A, B , C , D , E 是被末知节点监听到的信 标节点。)

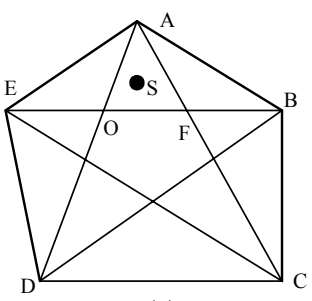

(a)

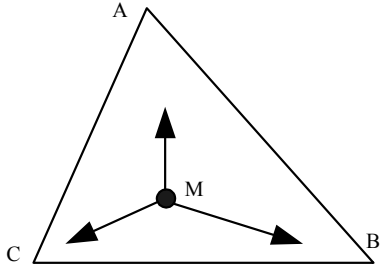

(b)
圖 2. APIT 定位原理圖.

Figure 2. The schematic diagram of APIT algorithm.

APIT 算法采用近似三角形内点测试法判定信标节 点构成的三角形是否包含该未知节点。如图 2 (b) 所 示, 在一个二维的平面上, 如果存在某个方向, 在这 个方向上移动 $M, M$ 跟 $A, B, C$ 的距离同时增大或变 小, 那么说明 $M$ 点在 $\triangle A B C$ 的外部, 反之, 则 $M$ 点在 $\triangle A B C$ 的内部。

仿真显示, APIT 测试错误率相对较小, 最坏情况 下为 $14 \%$ ，平均定位误差小于 $40 \%$ [5]。

APIT 算法对硬件要求较低, 且能耗较少, 但未知 节点必须直接与信标节点通信, 要求很高的信标节点 密度, 同时也与信标节点的分布有关。

\subsubsection{DV- H op 算法}

DV- Hop 算法的核心思想是未知节点通过路由方 法获得与信标节点之间最小跳数, 并计算平均每跳距 离, 以最少跳数和平均每跳距离的乘积作为末知节点 和信标节点之间的估计距离。最后利用三边测量法或 极大似然估计法计算未知节点坐标。

DV- Hop 定位过程分以下三个阶段 :

1) 计算与信标节点的最小跳数 : 信标节点向邻居 节点广播自身信息的分组，其中包括位置和跳数，初 始值为 0 。接收节点记录到每个信标节点的最小跳数, 然后将跳数值加 1 , 并转发给邻居节点。通过这个方 法, 网络中的所有节点都能够记录下到每个信标节点 的最小跳数。如图 $3, A$ 到 $B$ 的最小跳数为 6 。

2) 计算与信标节点的实际跳段距离 : 每个信标节 点根据第一个阶段中记录的其它信标节点的位置信息 和相距最小跳数, 利用式(2)估算平均每跳距离 :

$$
\text { HopSize }=\frac{\sum_{j \neq 1} \sqrt{\left(x_{i}-x_{j}\right)^{2}+\left(y_{i}+y_{j}\right)^{2}}}{\sum_{j \neq i} h_{j}}
$$

其中 $\left(X_{i}, Y_{i}\right) 、\left(X_{j}, Y_{j}\right)$ 是信标节点 $i 、 j$ 的坐标， $h_{j}$ 是信标节点 $\mathrm{i}$ 与 $\mathrm{j}$ 之间的跳数。然后, 信标节点将计 算的平均每跳距离用带有生存期字段的分组广播至网 


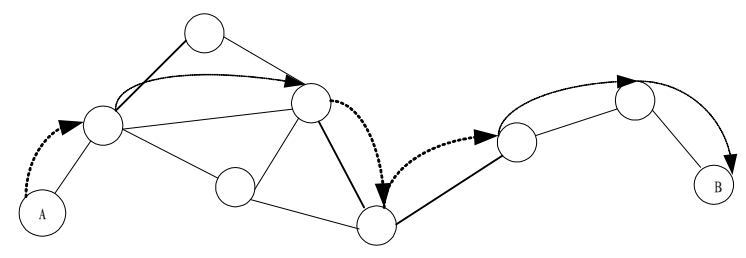

圖 3. DV-Hop 定位算法原理圖

Figure 3. The schematic diagram of DV-HOP algorithm.

络中，未知节点仅记录接收到的第一个平均每跳距 离, 并转发给邻居节点, 未知节点接收到平均每跳距 离后, 根据记录的最小跳数, 计算到每个信标节点的 跳段距离。

3) 计算自身坐标 : 未知节点利用第二阶段中记录 的到各个信标节点的跳段距离, 利用三边测量法或极 大似然估计法计算自身坐标。

DV- Hop 算法在网络平均连通度为 10 , 信标节点 比例为 $10 \%$ 的各向同性网络配置下, 定位精度为 $33 \%[10,11]$ 。

DV- Hop 算法对节点的硬件要求低, 实现简单。 其缺点是利用跳段距离代替直线距离, 存在一定的误 差。并且只能在各向同性的网络中表现良好, 因为只 有在各向同性的网络中平均跳距才能比较合理地估计 节点之间的实际距离。

\subsubsection{A morphous算法}

该算法与 DV- hop 类似, 都是采用相同的方法获 得距信标节点的跳数, 与 DV-Hop 不同的是, 它假设 网络平均连通度已知, 在网络部署前离线计算平均每 跳距离 HopSize。当获得三个或更多信标节点的梯度值 后, 未知节点使用 Sx HopSize 计算与每个信标节点距 离, 其中 $S$ 为某个信标节点的局部跳数平均值。如式 (3)，nbrs(i)表示节点所有邻居节点。

$$
S_{i}=\frac{\sum_{j=n b r s} h_{j}+h_{i}}{|n b r s(i)|+1}-0.5
$$

最后使用三边定位或极大似然估计法估算自身位 置。该算法需要预知网络平均连通度和较高的节点密 度, 并且算法将节点的通信半径作为平均每跳段距 离, 定位误差大。

\subsection{5. 凸规划定位算法}

凸规划算法的基本思想是 : 将节点间的通信连接 视为节点位置的几何约束, 把整个网络模型化为一个 凸集, 从而将节点定位问题转化为凸约束优化问题,
然后使用线性规划和半定规划方法得到一个全局优化 的解决方案, 确定节点位置[1]。如图 4 所示, 根据末 知节点与信标节点之间的通信连接和节点的射程, 得 出可能存在的区域(图中方块部分), 然后把矩形的质心 作为末知节点的估计位置。

凸规划是一种集中式定位算法, 在信标节点比例 为 $10 \%$ 的条件下, 定位精度大约为 $100 \%$, 为了保证凸 规划算法高效工作, 信标节点需放置在网络边缘, 否 则节点的位置估算会向网络中心偏移[1] 文献[12]中提 出了将节点间没有通信连接但同样视为节点位置约束 的思想来提高定位精度。

\subsubsection{MAP 算法}

MAP算法是 Kuo- Feng Ss等人提出的利用移动信标 节点的无需测距的定位算法。该算法的理论基础是圆的 任意两条不同弦的垂直平分线的交点即为圆心 在 MAP 中, 每个可移动信标节点在监测区域内移动并周期性地 广播信标信息，包括其 ID、位置和时间翟。其通信范围 内的未知节点接收并检查这些信息, 确定该导标信息是 否被作为一个信标节点。在获得至少三个信标点后就可 以形成两条以上的弦。进而计算圆心坐标。

MAP 算法定位精度高于其它无需测距的定位算 法。有较强的分布性、可扩展性和能量有效性。

\subsection{7. 其它定位算法}

Bounding Box 算法, 它定义了一个离散通信模型, 它假设节点通信范围是以自己为中心、二倍通信半径为 边长的正方形。如果一个节点能够与 $N$ 个信标节点通 信，则该节点在这 $N$ 个信标节点的正方形通信区域的交 叠矩形区域中。该算法的通信量和计算量很小，而且只 接收消息, 接收比发送消息耗能低, 符合传感器网络低 能耗的要求, 而且扩展性好。但它受到信标节点密度的 限制，定位精度严重依赖于信标节点的密度和分布。

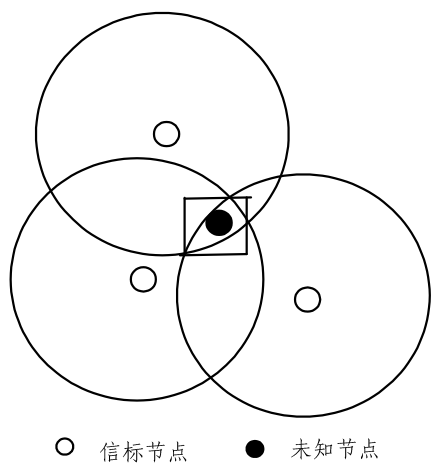

图 4. 节点位置几何约束图.

Figure 4. Geometric constraint graph of node position. 
APS 算法为利用距离矢量路由和 GPS 定位的原理 提出了一系列分布式定位算法。除前面介绍的 DV- hop 外还包括：DV-Distance DV-Bearing 和 DV-Radial DV- coordinate Euclidean。

DV- Distance 与 DV- Hop 算法所不同的是, 相邻节 点使用 RSSI 测量节点间距离, 然后利用类似于距离矢 量路由的方法传播与信标节点的累计距离。当未知节 点获得 3 个或更多信标节点的距离后使用三边定位法 定位。

Euclidean 定位算法运用了四边形边长和对角线存 在的约束关系, 得出了计算与信标节点相隔两跳的未 知节点位置的方法。

DV- Coordinate 算法, 利用 Euclidean 算法计算两 跳以内的邻近节点的距离, 建立局部坐标系统, 然后 相邻节点交换信息, 并将其转化为自身坐标系统中的 坐标, 再使用这些距离进行三边法测量定位。

DV- Bearing 和 DV- Radial 定位算法的思想是以逐 跳方式跨越两跳甚至三跳来计算与信标节点的相对角 度, 最后使用三角测量定位法。区别在于 DV- Radial 中的信标节点都安装有指南针, 从而可以获得绝对角
度信息, 并达到减少通信量和提高定位精度的目的。 实验显示, DV- Bearing 和 DV- Radial 算法在网络平均 连通度为 10.5 , 信标节点比例为 $20 \%, A O A$ 测量误差 小于 $5^{\circ}$ 的条件下，90\%以上的节点可以实现定位，定 位精度分别为 $40 \%$ 和 $25 \%$ [13]。

MDS MAP 定位算法采用的是多维定标数据分析 技术, 可在 range free 和 range based,2种情况下运行， 并可根据情况可实现相对定位和绝对定位。实验显 示, 当网络的节点密度减小时, 定位误差会增大, 并 且无法定位的节点越多; 而当网络连通度达到 12.2 时，几乎全部节点都可实现定位; 在拥有信标节点比 例 $2 \%$ 、平均连通度为 12.1 的网络中，在 range free 条 件下，定位误差约为 30\% ; 而在 range based 条件下， 定位误差为 $16 \%[14]$ 。

总体上，无需测距的定位算法无需利用额外硬 件实现节点间的测距，降低了对节点硬件要求较 低, 受环境影响也较小，但相对于基于测距的定位 算法而言定位误差较大, 并且对信标节点的密度和 分布有较高的要求。较适合于对定位精度要求不高 的大范围定位。

表 1 几种典型定位算法的比较.

Table 1. The comparison of several location algorithms.

\begin{tabular}{|c|c|c|c|c|c|}
\hline 分类 & 算法 & 优点 & 缺点 & $\begin{array}{l}\text { 信标节点 } \\
\text { 密度(个网) }\end{array}$ & 适用范围 \\
\hline \multirow{5}{*}{$\begin{array}{l}\text { 基于 } \\
\text { 测 距 } \\
\text { 的定 } \\
\text { 位算 } \\
\text { 法 }\end{array}$} & $\begin{array}{l}\text { 基于TOA 的定位 } \\
\text { 算法 }\end{array}$ & 定位较准确，算法简单，容易实现 & $\begin{array}{l}\text { 必须维持时钟同步, 需声波收发器, } \\
\text { 受多径效应影响 }\end{array}$ & $>3$ & $\begin{array}{l}\text { 需要精准定位的小型网络,多径效应 } \\
\text { 不明显的环境 }\end{array}$ \\
\hline & $\begin{array}{l}\text { 基于 TDOA 的定 } \\
\text { 位算法 }\end{array}$ & $\begin{array}{l}\text { 定位很准确, 只要外界建筑物没有 } \\
\text { 明显变化, 此法基本不受外界干 } \\
\text { 扰, 不需精确时钟同步 }\end{array}$ & $\begin{array}{l}\text { 受多径效应影响, 需超声波收发器, } \\
\text { 受到超声波传播距离的限制 }\end{array}$ & $>3$ & $\begin{array}{l}\text { 适应于小规模、需要较高定位精度的 } \\
\text { 环境 }\end{array}$ \\
\hline & $\begin{array}{l}\text { 基于 AOA 的 } \\
\text { 定位算法 }\end{array}$ & $\begin{array}{l}\text { 定位很准确, 还能提供节点的方位 } \\
\text { 信息 }\end{array}$ & $\begin{array}{l}\text { 易受外界环境影响, 需超声波收发 } \\
\text { 器, 功耗大 , 不适宜大规模网络 }\end{array}$ & $>3$ & $\begin{array}{l}\text { 需准确定位, 外界环境简单的小型网 } \\
\text { 络 }\end{array}$ \\
\hline & $\begin{array}{l}\text { 基于 RSSI 的 } \\
\text { 定位算法 }\end{array}$ & $\begin{array}{l}\text { 原理简单, 算法易实现, 不增加额 } \\
\text { 外硬件 , 有利节点的微型化, 可穿 } \\
\text { 透障碍物 }\end{array}$ & $\begin{array}{l}\text { 易受外界环境干扰 (电磁)，信号 } \\
\text { 强度有不同程度衰变, 定位粗糙 }\end{array}$ & $>3$ & $\begin{array}{l}\text { 外界环境不复杂, 对精度要求不高定 } \\
\text { 位, 如城市公交系统 }\end{array}$ \\
\hline & 质心定位算法 & $\begin{array}{l}\text { 算法简单易实现, 无需节点之间的 } \\
\text { 协调 , 完全基于网络的连通性, 通 } \\
\text { 信开销最小 }\end{array}$ & $\begin{array}{l}\text { 定位精度一般, 所需信标节点的密 } \\
\text { 度很大, 所以投入费用较大, 对信 } \\
\text { 标节点的分布要求很高 }\end{array}$ & $>10$ & $\begin{array}{l}\text { 信标节点密度较大, 分布均匀且易布 } \\
\text { 署的精度要求不高的定位 }\end{array}$ \\
\hline \multirow{5}{*}{$\begin{array}{l}\text { 非 基 } \\
\text { 于 测 } \\
\text { 距 的 } \\
\text { 定 位 } \\
\text { 算法 }\end{array}$} & APIT 算法 & $\begin{array}{l}\text { 定位精度相对较高, 性能稳定, 通 } \\
\text { 信开销较小, 对信标节点的分布要 } \\
\text { 求较低 }\end{array}$ & $\begin{array}{l}\text { 对网络的联通性要求较高, 所需信 } \\
\text { 标节点的密度很大 }\end{array}$ & $>10$ & $\begin{array}{l}\text { 环境复杂信号传播不规则, 节点须随 } \\
\text { 机部署的环境 }\end{array}$ \\
\hline & DV-Hop算法 & $\begin{array}{l}\text { 定位精度良好, 对硬件要求低, 实 } \\
\text { 现相对简单 }\end{array}$ & $\begin{array}{l}\text { 通信开销最大, 所需信标节点密度 } \\
\text { 较大 }\end{array}$ & $>8$ & $\begin{array}{l}\text { 适用于大规模的, 信标节点密度较 } \\
\text { 大, 可人工部署信标节点的环境 }\end{array}$ \\
\hline & Amorphous & 定位精度良好，对硬件要求低 & $\begin{array}{l}\text { 通信开销较大, 需预知网络平均连 } \\
\text { 通度, 需较高的节点密度 }\end{array}$ & $>8$ & $\begin{array}{l}\text { 规模较大, 网络平均连通度已知的环 } \\
\text { 境 }\end{array}$ \\
\hline & 凸规划定位算法 & 对硬件要求非常低 & $\begin{array}{l}\text { 定位精度低, 信标节点需部署在网 } \\
\text { 络边缘 }\end{array}$ & $>3$ & $\begin{array}{l}\text { 人工部署信标节点, 精度要求不高的 } \\
\text { 大规模网络 }\end{array}$ \\
\hline & MAP 算法 & $\begin{array}{l}\text { 定位精度高于其他非基于测距的 } \\
\text { 定位算法, 通信开销较小 }\end{array}$ & $\begin{array}{l}\text { 需有足够的能量以支持在监测区域 } \\
\text { 移动和广播信息 }\end{array}$ & $>3$ & $\begin{array}{l}\text { 精度要求高, 信标节点移动自定位的 } \\
\text { 网络 }\end{array}$ \\
\hline
\end{tabular}




\section{4. 几种典型定位算法的性能比较}

由以上对定位算法的分析可见, 在 WSN 的各种 定位算法中没有一种既能有效减少通信开销、降低 硬件要求并同时又能获得较高的定位精度和定位覆 盖率的算法, 没有普遍适合于各种应用的定位算 法, 它们各有优势和不足。因此, 根据以上对定位 算法的分析, 文章主要从定位精度、信标节点密度 和硬件要求等几个重要的性能评价指标的角度得出 了几种典型定位算法的优缺点, 进而得出其适用范 围(如表 1)。

\section{5. 结语}

无线传感器网络定位技术分为基于测距的定位算 法和无需测距的定位算法两大类。文章首先阐述了定 位算法的性能评价指标, 重点对目前几种典型定位算 法存在的基本问题和特点做了系统的分析比较，同时 对新生的定位算法进行了系统的阐述，研究发现定位 算法各有优势与不足, 没有普遍适合于各种应用的算 法, 需要通过综合考虑节点的规模、成本、功耗及对 定位精度要求来选择合适算法。因此文章针对以上问 题, 对典型定位算法的性能进行了评价, 得出了几种 典型定位算法各自的优势、不足和适用范围。

无线传感器网络定位技术需要进一步解决的问题 有 :

1) 定位算法性能评价的模型化和量化 ;

2) 算法的结合，使其能够优势融合，提出更佳的 定位算法 ;

3) 具有自适应(能根据环境及网络部署情况, 自动 选择合适的定位算法)功能的定位算法 ;

4) 建立自身定位仿真系统模拟定位算法的实现。

\section{References}

[1] F. B. Wang, L. Shi, and F. Y. Ren, "Self-localization systems and algorithms for wireless sensor networks [J]," Chinese Journal of software, Vol. 16, No. 5, pp. 857-868, 2005.

[2] Y. Qiu, C. C. Zhao, G. Dai, and C. J. Hu, "Research on localization technology for wireless sensor networks [J]," Computer Science, Vol. 5, No. 35, pp. 47-50, 2008.
[3] T. He, C. D. Huang, and B. M. Blum, "Range-free localization schemes in large scale sensor networks [C]," Proceedings of the 9th Annual International Conference on Mobile Computing and Networking, New York (NY, USA), pp. 81-95, 2003.

[4] X. H. Liu, F. M. Li, and P. Zheng, "A distributed and range-free node localization algorithm in wireless sensor network," in Chinese Journal of Sensors and Actuators, Vol. 21, No. 1, pp. 154-157, 2008.

[5] X. T. Zhang, X. J. Ban, S. H. Duan, et al., "Wireless sensor network and artifical life [M]," National Defense Industy Press, Beijing, 2008.

[6] J. Beutel, Geolocation in a PicoRadio environment [MS. Thesis], UC Berkeley, Berkeley, 1999.

[7] C. Savarese, J. M. Rabaey, and J. Beutel, "Locationing in distributed ad-hoc wireless sensor network," In Proceedings of the 2001 IEEE Int'l Conf.on Acoustics, Speech, and Signal, IEEE Signal Processing Society, Salt Lake, Vol. 4, pp. 2037-2040, 2001.

[8] S. Meguerdichian, S. Slijepcevic, V. Karayan, and M. Potkonjak, "Localized algorithms in wireless ad-hoc networks: Location discovery and sensor exposure." In Proceedings of the 2nd ACM Int'l Symp, on Mobile Ad Hoc Networking \& Computing, ACM Press, Long Beach, pp. 106-116, 2001

[9] S. N. Enrique, V. Vicekanandan, and W. S. V. Wong, "Dual and mixture monte carol localization algorithms for mobile wireless sensor networks [C]," IEEE Wireless Communications and Networking Conference, IEEE Press, Hong Kong, pp. 317-328, 2007.

[10] D. Nicolescu and B. Nath, "Ad-Hoc positioning systems (APS," In Proceedings of the 2001 IEEE Global Telecommunications Conference, IEEE Communications Society, San Antonio, Vol. 5, pp. 2926-2931, 2001.

[11] D. Niculescu and B. Nath, "DV based positioning in ad hoc networks," Journal of Telecommunication Systems, Vol. 22(1/4), pp. 267-280, 2003.

[12] N. Sundaram and P. Ramanathan, "Connectivity based location estimation scheme for wireless ad hoc networks," In Proceedings of the 2002 IEEE Global Telecom munications Conference, IEEE Communications Society, Taipei, Vol. 1, pp. 143-147, 2002.

[13] D. Niculescu and B. Nath, "Ad hoc positioning system (APS) using AoA," In Proceedings of the IEEE INFOCOM 2003, IEEE Computer and Communications Societies, San Francisco, Vol. 3, pp. 1734-1743, 2003.

[14] Y. Shang, W. Ruml, Y. Zhang, and Fromherz MPJ, "Localization from mere connectivity," In Proceedings of the 4th ACM Internatioal Symposium on Mobile Ad Hoc Networking \& Computing, ACM Press, Annapolis, pp. 201-212, 2003. 